\title{
Obstetric care for women that use antidepressants in pregnancy
}

\author{
Line Kolding ${ }^{1}$, Vera Ehrenstein ${ }^{1}$, Lars Pedersen ${ }^{2}$, Puk Sandager ${ }^{2}$, Olav Petersen ${ }^{3}$, Niels \\ Uldbjerg $^{1}$, and Lars Pedersen ${ }^{2}$ \\ ${ }^{1}$ Aarhus University \\ ${ }^{2}$ Aarhus University Hospital \\ ${ }^{3}$ Copenhagen University Hospital Rigshospitalet
}

September 25, 2021

Obstetric care for women that use antidepressants in pregnancy

Line Kolding, MD, $\mathrm{PhD}$

Vera Ehrenstein, MPH, DSc, Professor

Lars Pedersen, MSc, PhD, Professor

Puk Sandager, MD, PhD, Associate Professor

Olav B. Petersen, MD, PhD, Professor

Niels Uldbjerg, MD, DMSc, Professor

Lars H. Pedersen, MD, PhD, Professor

Corresponding:

Lars Henning Pedersen

Aarhus University Hospital / Aarhus University

Palle Juul-Jensens Blvd. 99, 8200 Aarhus N, Denmark

Email: lhp@clin.au.dk

Phone: +4550526512

We are grateful to Drs. Braillon and Bewley for their interest in our recent paper in the BJOG ${ }^{1}$ and would like to elaborate on some of the important points they raise.

We agree with Braillon and Bewley on the urgent need for improved pharmacovigilance of medication in pregnancy in general, and for antidepressants in particular. There are excellent international collaborations (e.g., the EuroCat) and local initiatives (e.g., the Swedish JanusInfo), but clinically we're often forced to rely on very limited information indeed. Systematic international recording as suggested by Braillon and Bewley would represent an important step forward.

On a smaller scale, we are establishing an automated surveillance system based on curated data that include information on both pre- and postnatally diagnosed malformations. We have, however, faced substantial legal and bureaucratic challenges, and have been forced to use data from the Central Denmark Region only, instead of national data. The surveillance system is consequently based on information on approx. 75,000 pregnancies, and even though it has the potential to aide clinical management, it is a drop in the ocean of the huge potential of for instance a comparable European collaboration. 
In our study, we used [?]2 redeemed prescriptions to define exposure with a prevalence $1.1 \% .{ }^{1}$ The prevalence of pregnant women that redeemed [?]1 prescription was 3.2\% (p. 3/ Table S1), and even though this is likely an overestimation due to non-adherence, the estimates are in line with previously reported prevalences in Scandinavia. ${ }^{2}$

Braillon and Bewley emphasise the need to also consider non-pharmacological treatment of some pregnant women with depression and, further, to provide evidence-based and individualised treatment of women in the reproductive ages. Optimal individualised care will definitely result in non-pharmacological treatment of some pregnant women but, reversely, will cause yet other women to continue or initiate pharmacological treatment. This is in line with what is almost a truism in this field, that the potential harmful foetal effects must be balanced against the potential benefits of a pharmacological treatment, but it is no easy task. Pregnant women might overestimate the foetal risks associated with use of medication ${ }^{3}$ and discontinue important treatment, on the other hand some may use medication when there may be a better alternative for them. Regardless, we need to provide optimal obstetric care for the pregnant women that choose treatment with antidepressants. If our results are correct, prenatal follow-up of pregnant women treated with venlafaxine may include targeted foetal heart scans, even though the underlying causal explanation for the observed association with cardiac malformations is undetermined.

1. Kolding L, Ehrenstein V, Pedersen L, Sandager P, Petersen OB, Uldbjerg N, et al. Antidepressant use in pregnancy and severe cardiac malformations: Danish register-based study. BJOG. 2021 May 25.

2. Zoega H, Kieler H, Norgaard M, Furu K, Valdimarsdottir U, Brandt L, et al. Use of SSRI and SNRI Antidepressants during Pregnancy: A Population-Based Study from Denmark, Iceland, Norway and Sweden. PLoS One. 2015;10(12):e0144474.

3. Wolgast E, Lindh-Åstrand L, Lilliecreutz C. Women's perceptions of medication use during pregnancy and breastfeeding - A Swedish cross-sectional questionnaire study. Acta Obstetricia et Gynecologica Scandinavica. 2019;98(7):856-64. 